\title{
The Effects of Horticultural Therapy Using Lavandula stoechas on Vital Signs, Pain, and Sleep Satisfaction of Hospice Patients
}

\author{
Eun Hee Ra, Suk Young Yun*, and Byung Jin Choi \\ Department of Horticulture, Daegu Catholic University, Gyeongsan 38430, South Korea
}

\begin{abstract}
The purpose of this study is to examine the effect of horticultural therapy utilizing Lavandula stoechas on vital signs, pain and sleep satisfaction of hospice patients in order to maintain the quality of life of hospice patients. Elderly patients with terminal cancer participated in horticultural therapy programs using L. stoechas plants designed so patients could inhale the scent of plants through various activities by cutting, rubbing, or stroking L. stoechas plants. To investigate the effect of horticultural therapy, the changes of vital signs such as blood pressure and pulse and pain intensities were measured and compared before and after each session, and sleep satisfaction was measured for the previous night's satisfaction after each session. As a result, the experimental group showed a statistically significant decrease $(p=.005)$ in pain intensity after the program and showed no significant difference in vital signs. The average sleep satisfaction of the control group was relatively low $(6.5 \pm 0.3)$, but sleep satisfaction of the experimental group was $9.0 \pm 0.6$, showing a statistically significant difference between the two groups $(p=.005)$. For hospice care, L. stoechas plants have applications in relieving pain and improving sleep satisfaction for terminally ill patients.
\end{abstract}

Keywords: blood pressure, hospice care, pulse, ward

\section{Introduction}

Death is the fear and sadness that everyone experiences in life, and hospice patients with terminal cancer suffer from confusion and pain in the body and mind, sleep imbalance, etc., which decreases the quality of life. Hospice care is caring patients with terminal cancer, focusing on palliative medicine that gives helps to patients in mental, spiritual, physical and social ways in order to maintain their dignity and quality of life (No et al., 1997). The physical and mental reaction that hospice patients with cancer pain fear the most is pain (Yang et al., 2009). The poor control of patients' pain can result in the lack of sleep, and thus cause anxiety and depression, which can be a serious problem that decreases the quality of life (Savard and Morin, 2001).

Horticultural therapy is the engagement of a person in various plant-based activities to improve the overall quality of life. Activities such as cutting and arranging flowers, trimming withered leaves and watering them were found to induce positive physiological changes in emotional functions (Yun and Choi, 2010). Alkaloids are compounds in herbs and are

Received: April 13, 2018, Revised: July 11, 2018, Accepted: July 17, 2018

First author: Eun Hee Ra, E-mail: naunhe@hanmail.net, ORCID: 0000-0003-1900-2340

*Corresponding author: Suk Young Yun, E-mail: yune1004@cu.ac.kr, ORCID: 0000-0002-5862-0021 
known to have the highest medicinal efficacy, among horticultural plants, for the nervous and digestive systems, liver, lungs, etc. (Park, 2003). In particular, the scent of Lavender was reported to be safe for humans and to have effects such as relieving psychological and physical stress and calming, and thus has been most widely used (Robins, 1999). Inhaling the scent was found to sedate and relax the central nervous system through an animal experiment and neurophysiological research method (Lis-Balchin and Hart, 1999). L. stoechas, used in this study, is one of 30 lavender species in the family Lamiaceae. It is a semi-evergreen shrub and is also called French lavender or Spanish lavender or Provence lavender. It grows up to a height of $30 \sim 60 \mathrm{~cm}$ and its gray, narrow-shaped leaves are about $1.5 \mathrm{~cm}$ long. The flowers are dark purple with two rabbit-ear-shaped bracts at the top of the spike (Cho, 2011). L. stoechas contains a large amount of camphor (53.3\%) as well as fenchone (24.3\%) and cineol (12.5\%) (Kim, 2000). In this regard, this study aimed to examine the effects of horticultural therapy using living herbs on hospice patients with terminal cancer by measuring changes in their vital signs (blood pressure and pulse), pain intensity and sleep satisfaction.

\section{Research Methods}

\section{Subjects}

The purpose of this study was explained to hospice patients with terminal cancer in a hospice ward located in D city, and their consent to participate in this experiment was obtained. Considering the characteristics of patients with terminal cancer, those who continued to participate in a total of 8 sessions were selected. For the final analysis, 10 subjects who did not want to participate in the program developed in this study were selected as the control group (76.8 \pm 13.3 years old) and 10 subjects who wanted to participate in the program were selected as the experimental group $(75.4 \pm 11.9$ years old). The general characteristics of the subjects are as shown in Table 1.

\section{Research Tools}

\section{Program}

This study aimed to examine the effects of a program in which subjects participate in activities while inhaling the scent of L. stoechas on the vital signs (blood pressure and pulse), pain intensity and sleep satisfaction of hospice patients with cancer pain. The program was provided for the experimental group between January and October, 2017, and a total of 8 sessions were provided. Each session was composed of various activities such as stroking, rubbing and cutting L. stoechas (Table 2), and was performed for 20 minutes between 2 and 4 p.m. everyday one to one. The program was performed on the bed of each subject in the experimental group, and Lavender plants as the outcome of horticultural activities were placed next to their bed for 24 hours in order to ensure they continue to smell the scent of Lavender. The program was led by a grade-1 welfare horticultural therapist (certified by the Korean Horticultural Therapy Association) and assisted by one nurse.

Table 1. General characteristics of subjects ( $N=20)$

\begin{tabular}{|c|c|c|c|c|c|c|c|}
\hline \multirow{2}{*}{ Group } & \multirow{2}{*}{$\mathrm{n}$} & \multicolumn{2}{|c|}{ Sex } & \multicolumn{2}{|c|}{ Spouse } & \multicolumn{2}{|c|}{ Religion } \\
\hline & & Male & Female & None & Have & None & Have \\
\hline Control & 10 & 8 & 2 & 2 & 8 & 4 & 6 \\
\hline Experimental & 10 & 7 & 3 & 5 & 5 & 1 & 9 \\
\hline
\end{tabular}


Table 2. Design of horticultural therapy program using L. stoechas

\begin{tabular}{clc}
\hline Session & \multicolumn{1}{c}{ Program } & Mindfulness instruction \\
\hline 1 & Decorating the flower pot & Looking into my current state \\
2 & Cutting and drying the leaves & Remembering old memories \\
3 & Making scent pouches & Faces reminded by different scents \\
4 & Flower net & Remembering happy memories \\
5 & Making wreaths & Memories to leave behind in the cycle of life \\
6 & Flower arrangement & Thinking of loved ones \\
7 & Flower bundles & Making a gift for myself \\
8 & Wall hangings & Accepting my current appearance \\
\hline
\end{tabular}

\section{Assessment Tools}

To identify the effects of the program, the pain intensity, blood pressure and pulse of the control group were measured two times per session at an interval of 20 minutes between 2 and 4 p.m., a total of 16 times during 8 sessions, and those of the experimental group were measured before and after horticultural therapy activities, a total of 16 times like the control group. The sleep satisfaction of the control group was measured on each day, a total of 8 times, and that of the experimental group was measured on the day of each session (8 sessions) after horticultural therapy activities. Blood pressure and pulse were measured by a nurse, and pain and sleep satisfaction were self-assessed by subjects. The assessment tool of each item is as follows:

\section{(1) Pain assessment tool}

Numeric rating scale (NRS) is a subjective pain scale $(10 \mathrm{~cm})$, used to rate pain, which is vertically divided into 10 parts and marked with numbers, and 0 means no pain; $1 \sim 3$, mild pain; $4 \sim 6$, moderate pain; and $7 \sim 10$, severe pain (Park and Kim, 2017).

\section{(2) Sleep assessment tool}

Subjective sleep satisfaction was measured using visual analogue scale (VAS) on the sleep of the previous day, and the higher the score, the higher sleep satisfaction. The scale is marked from 0 to 10 , and 0 means not at all satisfied; 5 , moderately satisfied; 10 , very satisfied (So, 2012).

\section{(3) Blood pressure and pulse assessment tools}

Blood Pressure Monitor UA-767JP (A\&D Medical, Japan), a digital blood pressure monitor, was used. When the systolic and diastolic blood pressure is $100 \sim 140 \mathrm{mmHg}$ and $60 \sim 90 \mathrm{mmHg}$ respectively, and the pulse rate is $50 \sim 95$, the conditions of subjects were considered normal.

\section{Data Analysis}

The vital signs and pain intensity of subjects measured in each session were categorized into pre-session and post-session, and their average value was calculated to examine the effectiveness of the horticultural therapy program. Non-parametric analysis was performed using IBM SPSS Program (19.0). Preliminary homogeneity between the control and experimental groups and changes in sleep satisfaction were analyzed using the Mann-Whitney test, and the results of 
vital signs and pain measured before and after activities were analyzed using the Wilcoxon signed rank test.

\section{Results and Discussion}

\section{Preliminary Homogeneity Test}

Preliminary homogeneity between the control and experimental groups were analyzed using the Mann-Whitney test, and there was no statistically significant difference in all the items measured in this study including vital signs (systolic blood pressure, $p=.406$; diastolic blood pressure, $p=.496$; and pulse, $p=.821$ ) and pain intensity ( $p=.139$ ), indicating that they were homogeneous (Table 3).

\section{Changes Before and After the Program}

\section{Changes in vital signs (blood pressure and pulse)}

Vital signs measured in this study include blood pressure and pulse. First, there was no statistically significant difference in the systolic blood pressure $(p=.169)$ and diastolic blood pressure $(p=.541)$ of the control group, but there was a statistically significant decrease from 78.9 to 78.2 in the pulse of the control group ( $p=.045$ ). Meanwhile, there was no statistically significant difference in the systolic blood pressure ( $p=.333)$, diastolic blood pressure $(p=.202)$, and pulse ( $p=.097$ ) of the experimental group (Table 4). These results coincide with the results of an earlier study that there was no statistically significant difference in the blood pressure of hospice patients after inhaling aroma (Louis and Kowalski, 2002). The reason why there was no significant difference in the vital signs of the experimental groups as the results above is that different factors involved in vital signs depending on the characteristics of oil are very complicated (Oh and Jung, 2002).

Table 3. Preliminary homogeneity test of vital signs in the control and experimental groups

\begin{tabular}{llcccc}
\hline Item & & Unit & Control & Experimental & $p$ \\
\hline \multirow{2}{*}{ Vital signs } & Systolic blood pressure & $\mathrm{mmHg}$ & 114.3 & 108.5 & $.406^{\mathrm{NS}}$ \\
& Diastolic blood pressure & $\mathrm{mmHg}$ & 69.2 & 72.8 & $.496^{\mathrm{NS}}$ \\
\multirow{2}{*}{ Pain } & Pulse & Beats/min & 78.9 & 80.1 & $.821^{\mathrm{NS}}$ \\
Sleep satisfaction & Score $^{\mathrm{z}}$ & 3.1 & 3.5 & $.139^{\mathrm{NS}}$ \\
\hline
\end{tabular}

${ }^{\mathrm{z}}$ Pain intensity $(0 \sim 10)$

${ }^{\mathrm{y}}$ Visual analogue scale $(0 \sim 10)$

${ }^{\mathrm{NS}}$ Non significant at $p<.05$ by Mann-Whitney test.

Table 4. Before-after analysis of vital signs in the control and experimental group

\begin{tabular}{|c|c|c|c|c|c|c|c|}
\hline \multirow{2}{*}{ Item } & \multirow{2}{*}{ Unit } & \multicolumn{3}{|c|}{ Control } & \multicolumn{3}{|c|}{ Experimental } \\
\hline & & Before & After & $p$ & Before & After & $p$ \\
\hline SYS $^{\mathrm{Z}}$ & $\mathrm{mmHg}$ & 114.3 & 114.7 & $.169^{\mathrm{NS}}$ & 108.5 & 108.1 & $.333^{\mathrm{NS}}$ \\
\hline DIA $^{y}$ & $\mathrm{mmHg}$ & 69.2 & 69.4 & $.541^{\mathrm{NS}}$ & 72.8 & 70.7 & $.202^{\mathrm{NS}}$ \\
\hline Pulse & Beats/min & 78.9 & 78.2 & $.045^{*}$ & 80.1 & 78.7 & $.097^{\mathrm{NS}}$ \\
\hline
\end{tabular}

${ }^{\mathrm{z}}$ Systolic blood pressure

${ }^{\mathrm{y}}$ Diastolic blood pressure

${ }^{\mathrm{NS}}$ Non significant, ${ }^{*}$ Significant at $p<.05$ by Wilcoxon signed-rank test. 


\section{Changes in pain}

There was no statistically significant difference in the pain intensity of the control group $(p=1.000)$, while the experimental group showed a statistically significant decrease from 3.5 before the program to 1.2 after the program ( $p=.005$, Table 5). The scent of camphor contained in L. stoechas has been used as a substance for various types of medicines such as painkiller, anesthetic, disinfectant and heart stimulant (Cho and Chiang, 2001), and the results of this study can be attributed to its pain relieving effect. However, another study reported that concentration during a horticultural therapy program resulted in a diversion effect that made hospice patients forget the pain they had, showing positive results in terms of pain relieving (Kim et al., 2006). Like the results of the study, horticultural activities using Lavender were also arranged along with inhaling its scent in this study, and, in this process, the scent was breathed in through the skin during rubbing activities and run through the body through the lymphatic system, and the peripheral circulation of the scent through the nose delivers the scent to the brain (So, 2012), which seems to relieve pain. It will be necessary to carry out studies to compare activities using Lavender plants with those not using Lavender plants in order to identify specific factors that are effective in relieving pain.

\section{Changes in sleep satisfaction}

The sleep satisfaction of the control group was measured in each session, a total of 8 sessions, in order to examine changes, and that of the experimental group was measured after each session (a total of 8 sessions) of horticultural therapy using Lavender plants. The average sleep satisfaction of the control group before the program was $7.8 \pm 1.5$, and the average of 8 days was $6.5 \pm 0.3$. The average sleep satisfaction of the experimental group before the program was $8.9 \pm 0.6$, and that of 8 days when the program was provided was $9.0 \pm 0.6$. There were statistically significant differences in sleep satisfaction between the control and experimental groups ( $p=.005$, Fig. 1). In this study, Lavender plants, instead of Lavender essential oil, were used for activities such as rubbing, stroking and cutting plants, and touching plants seems to be effective in this study. These results are similar to the result of an earlier study that aroma massage was effective in increasing the sleep satisfaction of patients with hypertension (Ju, 2012), and the result of Lee et al. (2002) that 1 2 drops of Lavender essential oil to a pillow or blanket before bedtime were effective in improving the quality of sleep.

In this study, a horticultural therapy program using Lavender plants was provided for hospice patients with cancer pain in order to maintain the quality of life of hospice patients with terminal cancer, and changes in their vital signs (blood pressure and pulse), pain intensity and sleep satisfaction were measured. The program was not effective in terms of vital signs, but showed positive results such as relieving pain and improving sleep satisfaction. However, although this experiment was conducted among patients with terminal cancer, they have different types of cancer, and the administration of pain killers and pain relievers might act as an external factor. In addition, since this study compared changes before and after the horticultural therapy program (20 minutes per day for 8 days) only, it seems to be difficult to generalize the results of this study.

Table 5. Before-after analysis of pain in the control and experimental group

\begin{tabular}{|c|c|c|c|c|c|c|c|}
\hline \multirow{2}{*}{ Item } & \multirow{2}{*}{ Unit } & \multicolumn{3}{|c|}{ Control } & \multicolumn{3}{|c|}{ Experimental } \\
\hline & & Before & After & $p$ & Before & After & $p$ \\
\hline Pain & Score $^{z}$ & 3.2 & 3.2 & $1.000^{\mathrm{NS}}$ & 3.5 & 1.2 & $.005^{* *}$ \\
\hline
\end{tabular}

${ }^{\mathrm{z}}$ Pain intensity (0 10)

${ }^{\mathrm{NS}}$ Non significant, ${ }^{*}$ Significant at $p<.01$ by Wilcoxon signed-rank test. 


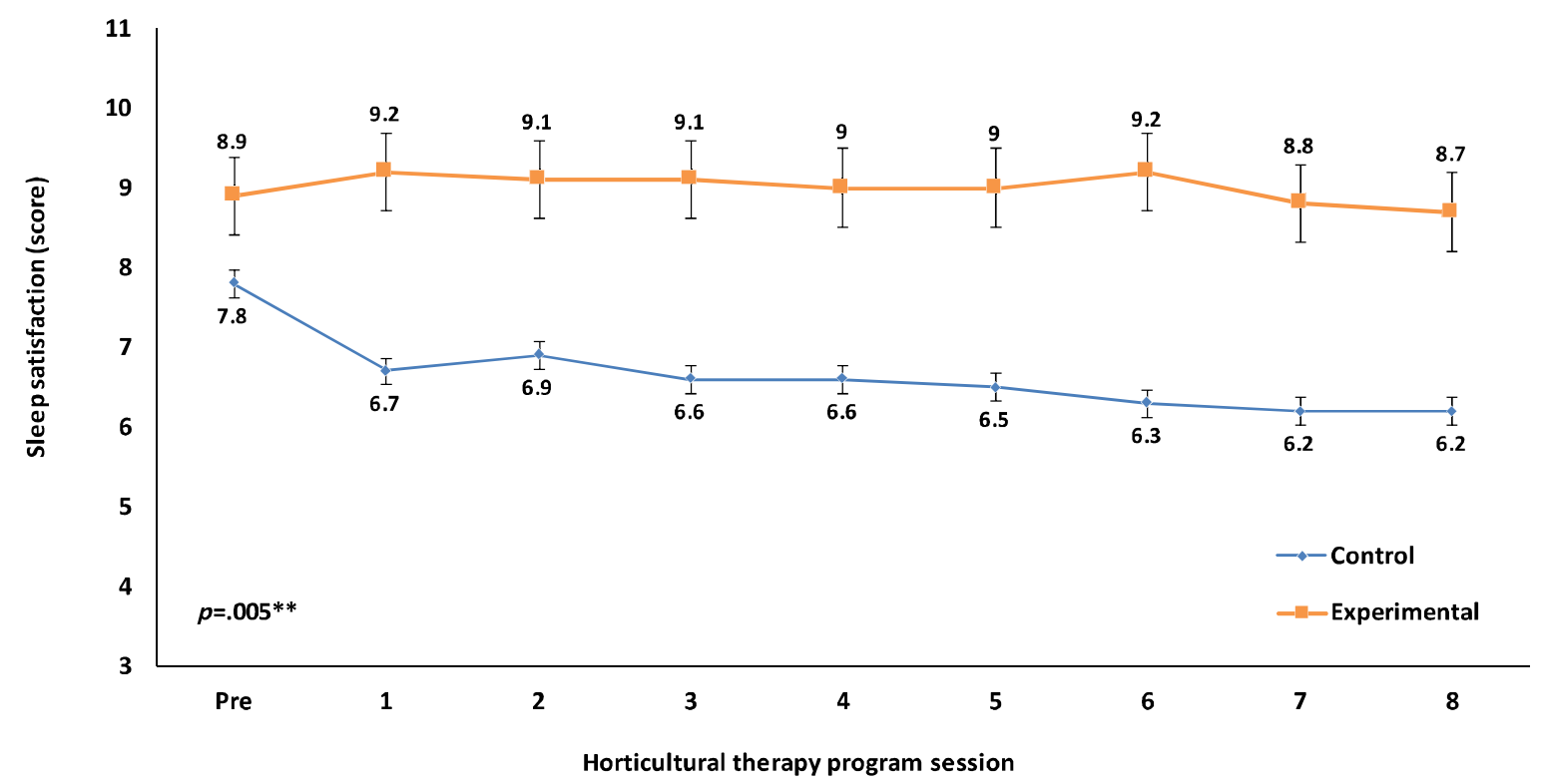

Figure 1. Changes in sleep satisfaction by session of the control and experimental groups. Error bars mean standard deviation. ${ }^{*}$ Significant at $p<.01$ by Mann-Whitney test.

\section{Conclusion}

This study aimed to examine the effects of horticultural therapy using Lavender plants on the vital signs, pain intensity and sleep satisfaction of hospice patients. L. stoechas plants, instead of Lavender essential oil, were used for activities in each session such as cutting, rubbing and stroking plants in their hospital room within the hospice ward. They participated in various activities of the horticultural therapy program and inhaled the scent of Lavender during the program. In order to identify the effects of the horticultural therapy program, changes in the vital signs (blood pressure and pulse) and pain intensity of the patients were measured before and after each session of the program (a total of 8 sessions), and their average values were calculated. Sleep satisfaction was measured on the day of each session after the program. There was a statistically significant decrease in the pulse of the control group $(p=.045)$ as well as in the pain intensity of the experimental group ( $p=.005$ ). The average sleep satisfaction of the control group was relatively low $(6.5 \pm 0.3)$, and that of the experimental group was $9.0 \pm 0.6$, showing a statistically significant difference between the two groups ( $p=.005$ ).

\section{References}

Cho, Y.H. and M.H. Chiang. 2001. Essential oil composition and antibacterial activity of Artemisia capillaries, Artemisia argyi, and Artemisia princeps. J. Korean Soc. Int. Agric. 13(4):313-320.

Cho, Y.J. 2011. Provence Lavender Load. Seoul, Korea: Culture Grapher by Ahn Graphics.

Ju, M.S. 2012. The effects of aroma massage on home blood pressure, ambulatory blood pressure and sleep for middle aged women with hypertension. Doctoral dissertation, Eulji University, Daejeon, Korea.

Kim, K.H., H.R. Lee, M.O. Song, S.H. Chung, and H.J. Chung. 2006. Effects of horticultural therapy program on serum cortisol, pain, anxiety and depression of the hospice patients. Korean J. Hortic. Sci. Technol. 24(1):95-103.

Kim, N.S. 2000. Determination of the lavender fragrances using solid phase trapping solvent extraction and GC-MSn. 
Master's thesis, Seoul Women's University, Seoul, Korea.

Lee, K.H., K.M. Park, and M.K. Ryu. 2002. The effect of aromatherapy with lavender essential oil on sleep disturbance and depression on middle-aged women. J. Korean Soc. Matern. Child Health 6(1):23-37.

Lis-Balchin, M. and S. Hart. 1999. Studies on the mode of action of the essential oil of lavender (Lavandula angustifolia P. Miller). Phytother. Res. 13(6):540-542.

Louis, M. and S.D. Kowalski. 2002. Use of aromatherapy with hospice patients to decrease pain, anxiety, and depression and to promote an increased sense of well-being. Am. J. Hosp. Palliat. Care 19(6):381-386. DOI: $10.1177 / 104990910201900607$

No, Y.J., S.S. Han, S.H. An, and C.G. Kim. 1997. Hospice \& Death. Seoul, Korea: Hyunmoonsa.

Oh, Y.H. and H.M. Jung. 2002. The effects of inhalation method using essential oils on the preoperative anxiety of hystrectomy patients. Korean J. Rehabil. Nurs. 5(1):18-26.

Park, G.W. 2003. Herb and Aromatherapy. Seoul, Korea: Sunjin printing.

Park, S.Y. and T.I. Kim. 2017. The effects of aroma inhalation on pain, anxiety, and heart rate variability among elderly women with total knee arthroplasty during continuous passive motion exercise. J. Korean Data Inf. Sci. Soc. 28(6):1383-1402.

Robins, J.L.W. 1999. The science and art of aromatherapy. J. Holist. Nurs. 17(1):5-17.

Savard, J. and C.M. Morin. 2001. Insomnia in the context of cancer: a review of a neglected problem. J. Clin. Oncol. 19(3):895-908.

So, H.R. 2012. The effect of aroma inhalation on pain, anxiety, vital sign and sleep of patients with colon resection. Master's thesis, Gachon University, Gyeonggi, Korea.

Yang, S.A., O.H. Cho, and Y.S. Yoo. 2009. A survey of cancer patients who visited emergency room. Korean J. Hosp. Palliat. Care 12(4):228-233.

Yun, S.Y. and B.J. Choi. 2010. Effect of horticultural therapy on the stress and serum cortisol of demented elders. Korean J. Hortic. Sci. Technol. 28(5):891-894. 
